\title{
Fraction auctions : the tradeoff between efficiency and running time
}

Citation for published version (APA):

Grigorieva, E., Herings, P. J. J., Müller, R. J., \& Vermeulen, A. J. (2012). Fraction auctions : the tradeoff between efficiency and running time. European Journal of Operational Research, 220(2), 577-587. https://doi.org/10.1016/j.ejor.2012.01.063

Document status and date:

Published: 01/01/2012

DOI:

10.1016/j.ejor.2012.01.063

Document Version:

Publisher's PDF, also known as Version of record

Document license:

Taverne

Please check the document version of this publication:

- A submitted manuscript is the version of the article upon submission and before peer-review. There can be important differences between the submitted version and the official published version of record.

People interested in the research are advised to contact the author for the final version of the publication, or visit the DOI to the publisher's website.

- The final author version and the galley proof are versions of the publication after peer review.

- The final published version features the final layout of the paper including the volume, issue and page numbers.

Link to publication

\footnotetext{
General rights rights.

- You may freely distribute the URL identifying the publication in the public portal. please follow below link for the End User Agreement:

www.umlib.nl/taverne-license

Take down policy

If you believe that this document breaches copyright please contact us at:

repository@maastrichtuniversity.nl

providing details and we will investigate your claim.
}

Copyright and moral rights for the publications made accessible in the public portal are retained by the authors and/or other copyright owners and it is a condition of accessing publications that users recognise and abide by the legal requirements associated with these

- Users may download and print one copy of any publication from the public portal for the purpose of private study or research.

- You may not further distribute the material or use it for any profit-making activity or commercial gain

If the publication is distributed under the terms of Article $25 \mathrm{fa}$ of the Dutch Copyright Act, indicated by the "Taverne" license above, 
Interfaces with Other Disciplines

\title{
Fraction auctions: The tradeoff between efficiency and running time
}

\author{
Elena Grigorieva ${ }^{\mathrm{b}, 1}$, P. Jean-Jacques Herings ${ }^{\mathrm{a}, 2}$, Rudolf Müller ${ }^{\mathrm{b}, *, 3}$, Dries Vermeulen ${ }^{\mathrm{b}, 4}$ \\ a Department of Economics, Maastricht University, P.O. Box 616, 6200 MD Maastricht, The Netherlands \\ ${ }^{\mathrm{b}}$ Department of Quantitative Economics, Maastricht University, P.O. Box 616, 6200 MD Maastricht, The Netherlands
}

\section{A R T I C L E I N F O}

Article history:

Received 8 March 2010

Accepted 31 January 2012

Available online 7 February 2012

\section{Keywords:}

Query auction

Efficiency

Running time

\begin{abstract}
A B S T R A C T
This paper studies the sales of a single indivisible object where bidders have continuous valuations. In Grigorieva et al. [14] it was shown that, in this setting, query auctions necessarily allocate inefficiently in equilibrium. In this paper we propose a new sequential auction, called the $c$-fraction auction. We show the existence of an ex-post equilibrium, called bluff equilibrium, in which bidders behave truthfully except for particular constellations of observed bids at which it is optimal to pretend a slightly higher valuation. We show c-fraction auctions guarantee approximate efficiency at any desired level of accuracy, independent of the number of bidders, when bidders choose to play the bluff equilibrium. We discuss the running time and the efficiency in the bluff equilibrium. We show that by changing the parameter $c$ of the auction we can trade off efficiency against running time.
\end{abstract}

(c) 2012 Elsevier B.V. All rights reserved.

\section{Introduction}

The English auction remains the predominant auction format used in practice, though we know since Vickrey [25] that it is strategically equivalent to the second-price, sealed bid auction. Rothkopf et al. [21] argued that third parties being able to "capture fractions of the economic rent revealed by the second price procedure" is one of the reasons why we hardly observe any Vickrey auctions in practice. Engelbrecht-Wiggans and Kahn [11] support this argument in their analysis. They study a model of a procurement auction where the winner of the auction might have to undergo a negotiation with a third party after the auction. In this negotiation, information of the third party about the winner's cost revealed in the auction can have a negative influence on the winner's surplus and the auctioneer's revenue. Also for combinatorial auctions-settings with multiple, heterogenous goods and bidder valuations for bundles of items-iterative auctions have been the most popular approach: first in form of simultaneous ascending auctions (Cramton [7]), later in form of ascending or descending

\footnotetext{
* Corresponding author. Tel.: +31 433883799.

E-mail addresses: p.herings@maastrichtuniversity.nl (P. Jean-Jacques Herings), r.muller@maastrichtuniversity.nl (R. Müller), d.vermeulen@maastrichtuniversity.nl (D. Vermeulen).

1 The author acknowledges support by the Dutch Science Foundation, NWO through grant 401-01-101.

2 The author acknowledges, support by the Dutch Science Foundation NWO through a VICI-grant.

3 The author acknowledges support by Hausdorff Institute of Mathematics, Bonn.

${ }^{4}$ The paper has greatly benefitted from the comments and suggestions of three anonymous referees.
}

combinatorial auctions (de Vries et al. [10] and Mishra and Veeramani [18]) and clock and clock-proxy auctions (Ausubel et al. [1]).

Most ascending auctions studied in the theoretical literature should be implemented by a continuous, increasing price clock, where bidders drop out whenever the price exceeds what they are willing to pay. However, in practice we see almost exclusively implementations of the following two variants: (1) a discrete clock is increased by increments chosen by the auctioneer; (2) bidders submit increasing bids which exceed the current high bid plus some minimum increment, usually in terms of a percentage of the standing high bid. Cramton [7] reports that "bid increments in the 5 to 10 percent range are required to get the auction to conclude in a manageable number of rounds". For example, if the auction is used to decide on the sales of radio spectrum, such increments are in the order of hundreds of millions of dollars, and can cause bidders to drop out because the next bid would exceed their valuation.

When the auctioneer sets bid increments he has to deal with a tradeoff between efficiency and running time of the auction. As bid increments define a decomposition of the continuum of valuations into intervals, a large bid increment might lead to an inefficient allocation of the item because it prohibits distinguishing between bidders whose valuations are in the same interval. Small increments decrease the chance of allocative inefficiency, but increase the running time of the auction, and thereby participation costs, substantially [9]. Despite its practical relevance, the tradeoff between these two goals has found very little attention in the academic literature. The furthest reaching theoretical evaluation of discrete bid levels has been given in David et al. [9], following up on [8]. They provide a recipe on how to set a finite number of discrete bid levels in order to maximize expected revenue of the 
auctioneer. Thereby they extend the analysis of Rothkopf and Harstad [22], which was limited to either 2 bidders or 2 bid levels. However, the recipe of David et al. can be solved analytically in a few cases only. In other cases, the auctioneer has to rely on numerical calculations. The key qualitative insight of these articles is that decreasing increments are preferable to constant or increasing bid increments-the common practice.

In this article we introduce a discrete query auction, called $c$ fraction auction, for the sale of a single item. The auction gives the auction designer full control of the tradeoff between inefficiency and running time. Based on a prior of bidders' valuations, the auctioneer can choose a single parameter, called $c$ throughout, to regulate both running time and expected inefficiency. The practical implementation requires the computation of values of the quasi-inverse of cumulative distribution functions as the only numerical tool. The auction is detail-free from the bidders' perspectives by offering them an ex-post Nash equilibrium, called bluff equilibrium, that differs only slightly from truth-telling (see Section 3 for details). In other words, the equilibrium analysis does not have to make assumptions about bidders' beliefs of other bidders' valuations.

Our main contribution is to provide a detailed analysis of the performance of the $c$-fraction auction under the so-called bluff equilibrium. First, we investigate the running time of the auction according to two measures, the expected number of rounds and the expected number of queries performed in the auction. ${ }^{5}$ For both measures, we first derive an exact recursive formula and then give an upper bound for the function defined by this formula. We prove that, for a fixed $c$, the expected number of rounds is bounded by a function that is logarithmic in the number of players, while the expected number of queries is bounded by a function that is linear in the number of players. We also give an impression of the number of rounds and number of queries by using computer simulations.

Second, we analyze the level of inefficiency of the auction. As measures of inefficiency, we employ the probability of inefficient allocation and the expected loss of welfare. For the probability of inefficient allocation, we prove that it is no more than $c$ for any number of players, again after deriving an exact recursive formula. This is remarkable because for an ascending auction with constant or increasing bid increments, and, say, uniform i.i.d. valuations, the probability of inefficient allocation is increasing in the number of bidders and converging to 1 . Indeed, the number $n_{\max }$ of bidders with a value in the largest interval is increasing, and as such an auction cannot distinguish between them, the probability of inefficient allocation is $\frac{n_{\max }-1}{n_{\max }}$. With respect to the expected loss of welfare, we derive for each distribution a constant $\gamma(c)$ such that the expected loss of welfare is bounded from above by $\gamma(c) c$ for any number of players. When valuations are uniformly drawn from $[0,1)$ it holds that $\gamma(c)=c$ and for the exponential distribution with parameter $\lambda$ we have that $\gamma(c)=-(\ln (1-c)) / \lambda$. Our results imply that by choosing the appropriate $c$, the minimum level of efficiency can be determined by the auctioneer before it is known how many players will participate in the auction. We also give an impression of the expected loss of welfare by using computer simulations. Furthermore, we show that there is a tradeoff between efficiency and running time: to increase the efficiency of the auction we have to pay by increasing the number of rounds and increasing the number of queries.

In Grigorieva et al. [14] we study the limitations of query auctions with respect to the objective of economic efficiency maximization in a setting with valuations distributed according to a continuous density function. We show that any ex-post

\footnotetext{
${ }^{5}$ As a query we consider each separate question of the auctioneer to an active player. As a round we consider a sequence of queries in which each active player is asked to act exactly once.
}

equilibrium in an ex-post individual rational query auction that ends with positive probability after a finite number of queries cannot be fully efficient. This result implies that in the setting of continuous valuations, full efficiency can only be achieved at the expense of an infinite running time of a query auction for almost all realizations of valuations. Our results on the $c$-fraction auction prove a counterpart of this negative result: for any $c>0$, the probability of inefficient allocation can be limited by $c$ using an individually rational query auction with an ex-post equilibrium that ends after a finite number of rounds for all realizations of valuations. Furthermore, the number of rounds is logarithmic in the number of bidders, and independent of the range of valuations.

Our paper is closely related to David et al. [9]. However, they focus on revenue maximization and touch upon efficiency losses only on the side by showing that revenue-optimal discrete bid levels achieve better economic efficiency than equidistant levels. Though the paper is more ambitious since it tries to solve the optimization problem, a practical implementation requires complex numerical calculations and at least a prior on the number of bidders. David et al. [9] provide a Bayesian machine learning model, based on closing prices of previous auctions, to compute revenue maximizing discrete bid levels when no ex-ante information on the number of bidders and their distribution of valuations is available.

The earliest paper on discrete bid levels in continuous settings that we could trace is by Chwe [4]. Chwe studies the impact on revenue when discrete bids rather than continuous bids are used in sealed-bid, first price auctions. He analyzes equilibrium bidding strategies and shows that revenue with discrete bids is always lower than with continuous bids. Yu [26] extends his equilibrium analysis to English, Vickrey, and Dutch auctions.

Another stream of literature studies iterative auctions from the viewpoint of preference elicitation. Determining one's valuation with a precision up to the last digit can be computationally demanding, see for example Larson and Sandholm [16], Parkes [20], and Sandholm [23]. In combinatorial auctions, the full revelation of agents' preferences may require a prohibitive amount of communication, see for example Nisan and Segal [19]. Such considerations lead to an interest in auctions where agents need not reveal their information entirely but only partially. One approach is to limit communication in a sealed bid auction to a finite number of possible bits, see Blumrosen et al. [2]. Another approach is incremental elicitation of valuations in multiple rounds. It has been recognized that multi-round mechanisms can reduce the amount of preferences that need to be revealed and reduce the amount of computation and communication, compared to single-round mechanisms advocated by the revelation principle, see Blumrosen et al. [3] and Conitzer and Sandholm [6]. Incremental elicitation of bidder valuations has been modeled by query auctions (see e.g., Conen and Sandholm [5]). In a query auction the auctioneer sequentially queries the agents about specific aspects of their preferences. As an answer to the query, an agent can choose one of a finite set of actions. Through incremental querying, the auctioneer gradually collects the information on agents' valuations. By using a query strategy in which previously revealed information guides the selection of subsequent queries, elicitation is targeted towards pertinent information. Incremental querying has been applied in different settings (see e.g. Conen and Sandholm [5] and Grigorieva et al. [13]) and it has been shown that only a small fraction of agents' valuation information needs to be revealed before the (approximately) optimal allocation can be determined (Grigorieva et al. [12], Hudson and Sandholm [15]).

When evaluating the effectiveness of elicitation we may generally care about the running time expressed by the number of queries that are required to determine an optimal allocation (Sandholm and Boutilier [24]). Since information about agents' valuations becomes more refined with each query, a higher 
number of queries leads to a better allocation. Our proposed $c$-fraction auction provides a framework that lets the auctioneer explicitly trade off the two goals.

The paper is organized as follows. Section 2 introduces the rules of the $c$-fraction auction and Section 3 derives what we call the bluff equilibrium. Section 4 addresses the probabilities by which bidders make yes and no responses. In Section 5, the running time of the auction is analyzed. Section 6 is devoted to the analysis of the efficiency of the auction and remarks about the tradeoff between running time and efficiency. Section 7 provides some concluding remarks.

\section{The $c$-fraction auction}

Suppose a single indivisible object is auctioned to a set $N=\{1, \ldots, n\}$ of players. The players have quasi-linear utilities. We assume independent private valuations drawn from a common continuous probability distribution with density $f$ and cumulative density $F$. The support of $f$ belongs to $\mathbb{R}_{+}$. The minimum of this support is denoted by $\alpha$ and the supremum by $\beta$, where we allow $\beta$ to be infinite and assume that $\beta$ strictly exceeds every possible valuation. ${ }^{6}$ Since $F$ may not be strictly increasing, it may not have an inverse. By $F^{-1}:[0,1) \rightarrow \mathbb{R}$ we denote the function such that $F^{-1}(y)=\max \left\{x \in \mathbb{R}_{+} \mid F(x)=y\right\}$.

Before the start of the auction there is a lottery that determines an ordering of the players. Without loss of generality we assume that this ordering is $1 \prec 2 \prec \cdots \prec n-1 \prec n$. A player with a lower ranking is called a predecessor.

The auction runs for a number of rounds. A round $r$ is characterized by a payment $p_{r}$, a query price $q_{r}$, an upper bound $u_{r}$, rand a set of active players $A_{r}$. The payment specifies the price to be paid if an active player wins in this round. The query price is used by the auctioneer to ask the active players whether their valuation is larger than or equal to the query price. Active players are queried publicly in increasing order-player $i$ before player $i+1-$ so that an active player can observe the bids of his predecessors. In each round the query price $q_{r}$ is chosen from the open interval $\left(p_{r}, u_{r}\right)$, where does not occur, i.e. at least two players remain active forever, the winner is determined according to the order of players: among those players who remain active the player with the highest ranking wins. The price the winner pays is equal to the limit of the sequence of payments $\left(p_{r}\right)_{r \in \mathbb{N}}$ that occur in the subsequent rounds in the auction. Since the sequence of payments is increasing, this limit is equal to the supremum of the payments, and is denoted by $p_{\infty}$.

For the $c$-fraction auction, where $c \in(0,1)$, the query price $q_{r}$ is chosen as the maximal $q$ for which

$\frac{F(q)-F\left(p_{r}\right)}{F\left(u_{r}\right)-F\left(p_{r}\right)}=c$

i.e. $c$ is equal to the probability that a valuation belongs to the interval $\left(p_{r}, q_{r}\right]$ conditional on this valuation being in the interval $\left(p_{r}, u_{r}\right]$. For the uniform distribution for example it holds that $q_{r}=p_{r}+c\left(u_{r}-p_{r}\right)$. Equivalently, we can define

$q_{r}=F^{-1}\left((1-c) F\left(p_{r}\right)+c F\left(u_{r}\right)\right)$.

Consider the following strategy for player $i$ having valuation $v_{i}$. This player says yes in round $r$ if and only if

1. $v_{i} \geqslant q_{r}$, or

2. $p_{r} \leqslant v_{i}<q_{r}$ and no active predecessor of $i$ said yes in round $r$.

In the second part of the definition, we mean by an active predecessor of $i$ in round $r$ a player in $A_{r}$ that is a predecessor of $i$. Because this part of the definition involves a certain amount of bluff, we call this strategy the bluff strategy. Formally, the auction is given by an extensive form game, the bluff strategy of player $i$ is a function from the set of decision nodes of player $i$ to $\{y e s, n o\}$, and is denoted by $b_{i}$. The following example illustrates how the auction proceeds when all players follow the bluff strategy.

Example 1. Suppose five players, with valuations uniformly distributed on $[0,1)$, participate in the $c$-fraction auction, where $c$ is equal to 0.5 . Players have the following private valuations: 0.43 , $0.71,0.38,0.79$, and 0.86 . The auction proceeds as follows.

\begin{tabular}{lllllllll}
\hline Round $r$ & Payment $p_{r}$ & Query price $q_{r}$ & Set of active players $A_{r}$ & Player 1 & Player 2 & Player 3 & Player 4 & Player 5 \\
\hline 1 & 0 & 0.5 & $\{1,2,3,4,5\}$ & yes & yes & no & yes & yes \\
2 & 0.5 & 0.75 & $\{1,2,4,5\}$ & no & yes & - & yes & yes \\
3 & 0.75 & 0.875 & $\{2,4,5\}$ & - & no & - & yes & no \\
\hline
\end{tabular}

$u_{r}$ is allowed to be infinite.

The initial set of active players is $A_{1}=N$. The auction starts with $p_{1}=\alpha, u_{1}=\beta$, and some $q_{1}$ in $\left(p_{1}, u_{1}\right)$. Given the current set $A_{r}$, the payment $p_{r}$, the query price $q_{r}$, the upper bound $u_{r}$, rand the bids of players in round $r$, the characteristics of the next round $r+1$ are defined as follows. If all active players submit a no bid, they all remain active, i.e. $A_{r+1}=A_{r}$, the payment remains the same, and the upper bound is set to the previous query price, i.e. $p_{r+1}=p_{r}$ and $u_{r+1}=q_{r}$. If at least two active players submit a yes bid, all players that said yes remain active, the upper bound remains the same, and the payment is set equal to the previous query price, i.e. $u_{r+1}=u_{r}$ and $p_{r+1}=q_{r}$. In both cases, as a function of the bounds, a new query price $q_{r+1}$ in $\left(p_{r+1}, u_{r+1}\right)$ is determined in the way specified below. If only one active player submits a yes bid, the auction stops, this player wins the auction, and pays $p_{r}$. If such a moment

\footnotetext{
${ }^{6}$ Requiring all valuations to be strictly smaller than $\beta$ is a mild, though non-standard, technical assumption that is helpful for some of our theorems. See also footnote 8 .
}

In the first round, player 1 , having no predecessor and having a valuation larger than $p_{1}$, says yes. Every other player, having now an active predecessor who said yes, says yes if and only if his valuation is greater than or equal to $q_{1}=0.5$. All players except player 3 say yes and therefore remain active. The payment and the query price increase to 0.5 and 0.75 , respectively. Since $v_{1}<p_{2}$ player 1 says no in the second round. Now player 2 has no active predecessor who said yes and since $v_{2} \geqslant p_{2}$ he says yes. Players 4 and 5 say yes since their valuations are larger than $q_{2}=0.75$. Again the payment and the query price increase. In the third round player 2 says no, player 4 , having now no active predecessors who said yes, says yes, rand player 5 says no. In this round there is only one yes decision, meaning that the auction ends. Player 4 wins the auction and pays 0.75 . 
Notice that the outcome in the example is not efficient-the winner is not the player with the highest valuation. Later in the paper we investigate how inefficient this auction is by analyzing the probability of an inefficient allocation and the expected loss of welfare.

\section{Bluff equilibria}

In this section we show that the profile of bluff strategies constitutes an ex-post individually rational ex-post equilibrium called the bluff equilibrium. An ex-post equilibrium is a strategy profile such that, given any realization of valuations, the plan of action prescribed to a bidder in the auction by his strategy is a best response to the plans of action prescribed by the strategies of the other bidders given their valuations. A strategy is ex-post individually rational if for every realization of valuations and for any profile of actions of the player's opponents, the strategy leads to non-negative utility.

\section{Proposition 3.1. The bluff strategy is ex-post individually rational.}

Proof. Consider an arbitrary strategy profile where player $i$ follows his bluff strategy. If $i$ is not the winner, then he has utility 0 . Thus assume that $i$ is the winner. Note that $i$ must be an active player in all rounds of the auction, as only active players can become winners. We show that $p_{r} \leqslant v_{i}$ in each round $r$, which implies that $i$ has positive utility. By construction, $p_{r} \leqslant v_{i}$ for $r=1$. Suppose the inequality holds up to round $r$. We show that it also holds in round $r+1$, or the auction ends in round $r$. There are two cases.

Case 1. A predecessor of $i$ says yes in round $r$. To become the winner, $i$ must say yes as well. As $i$ plays the bluff strategy, it holds that $v_{i} \geqslant q_{r}$. Furthermore, we have that $p_{r+1}=q_{r}$.

Case 2. No predecessor of $i$ says yes in round $r$.When $i$ says no, all successors of $i$ in $A_{r}$ must also say no for $i$ to be the winner, so $p_{r+1}=p_{r} \leqslant v_{i}$. When $i$ says yes and all successors say no, the auction ends. When $i$ says yes and one successor says yes, either $p_{r+1}=q_{r} \leqslant v_{i}$ or $p_{r+1}=q_{r}>v_{i}$. We show that the latter case contradicts the assumption that $i$ is the winner. Note that $i$ will say no in each future round $r^{\prime}$ where he is active, as $v_{i}<p_{r+1} \leqslant q_{r^{\prime}}$. When any other active player says yes in any future round $r^{\prime}, i$ will not be the winner. When all other active players-and there is at least one in round $r+1$-keep saying no, the set of active players stays the same in all future rounds. In this case an active player with higher rank than $i$ wins.

Theorem 3.2. The bluff strategy profile is an ex-post Nash equilibrium.

Proof. Let $v$ be a realization of valuations and $f_{i}$ be a strategy for player $i$, i.e. a function from the set of decision nodes for player $i$ to $\{y e s, n o\}$. We show that $f_{i}$ is not a profitable deviation from $b_{i}$ against $b_{-i}$. Let $h$ be the first decision node at which player $i$ following $f_{i}$ deviates from the bluff strategy, and let $r$ denote the corresponding round. (Obviously, if $f_{i}$ coincides with $b_{i}$ at all decision nodes, $f_{i}$ is not a profitable deviation.) Notice that since we treat $v$ as given and apart from $v$ there is no imperfect information, the node $h$ is well-defined. We consider two cases.

Case 1. Let $h$ be such that at least one predecessor of $i$ in $A_{r}$ has said yes. If $f_{i}(h)=$ no and $b_{i}(h)=y e s$, the payoff of playing $f_{i}$ is 0 , while according to Proposition 3.1 the payoff of playing the bluff strategy is at least 0 . Consider the case where $f_{i}(h)=$ yes and $b_{i}(h)=$ no. When player $i$ says yes in $h$, there are at least two players who say yes in round $r$. The winning payment will be at least $q_{r}$. Further, $v_{i}<q_{r}$ because $b_{i}(h)=$ no. Hence, the payoff of playing $f_{i}$ is non-positive while the payoff of playing $b_{i}$ is 0 .

Case 2. Let $h$ be such that none of the predecessors of $i$ in $A_{r}$ has said yes. If $f_{i}(h)=$ yes and $b_{i}(h)=n o$, we know that $v_{i}<p_{r}$. Since the payment of the winner is at least $p_{r}$, playing $f_{i}$ leads to a non-positive payoff, while playing according to $b_{i}$ guarantees a non-negative payoff. Consider the case where $f_{i}(h)=$ no and $b_{i}(h)=y e s$, so $v_{i} \geqslant p_{r}$. Suppose that $v$ is such that all successors of $i$ say no if $i$ says yes. Then, following $b_{i}$, player $i$ wins at a price $p_{r}$ while following $f_{i}$ he might win at a price at least $p_{r}$. Now suppose that $v$ is such that there is a successor $j$ of $i$ that says yes if player $i$ says yes. Since player $j$ uses the bluff strategy, he will also say yes when player $i$ switches to no. But then the payoff of playing $f_{i}$ would be 0 while the payoff of playing $b_{i}$ is non-negative.

Theorem 3.3. The allocation under the bluff equilibrium is not expost efficient.

Proof. See the example in Section 2.

We argue next that $c$-fraction auctions have a finite running time under the bluff equilibrium for any realization of valuations. Notice that this statement is stronger than just saying that we have a finite running time almost surely. We also argue that when every bidder plays according to his bluff strategy, in any round of the auction there is at least one player who says yes.

Theorem 3.4. The bluff equilibrium has a finite running time for every realization of valuations. Moreover, the query price increases from round to round up to the moment where the winner is found.

Proof. We first claim that the winner of the auction, say player $j$, says yes in every round of the auction. Suppose not, then let $r$ be the first round in which he says no. Now all players in $A_{r}$ say no in round $r$, since otherwise $j$ cannot be the winner of the auction. Either $r=1$ or in round $r-1$ player $j$ said yes and so did all players in $A_{r}$. If $r=1$, then $v_{j} \geqslant p_{1}=\alpha$, so player $j$ says yes, leading to a contradiction. If in round $r-1$ all players in $A_{r}$ said yes, then so did a player $i$ with a predecessor in $A_{r}$, implying that $v_{i} \geqslant q_{r-1}=p_{r}$. Observing a no from all his predecessors in round $r$, player $i$ should say yes in round $r$ when following the bluff strategy, a contradiction. We have shown that the winner of the auction says yes in every round of the auction. It then follows that the query price increases from round to round up to the moment where the winner is found.

Suppose the valuation $v$ is such that the running time of the auction is infinite. Since by the previous paragraph the query price increases from round to round, we have $p_{\infty}=\beta$. Since the winner of the auction, say player $j$, says yes in every round $r$, we have for all $r \in \mathbb{N}, v_{j} \geqslant p_{r}$, so $v_{j} \geqslant \beta$, ra contradiction to our assumption that $\beta$ strictly exceeds every possible valuation. ${ }^{7}$

As a corollary to Theorem 3.4 we find an easy characterization of the query price in round $r$.

\footnotetext{
7 Without the assumption that $\beta$ strictly exceeds every possible valuation, the running time would be infinite exactly when two or more bidders have valuation $\beta$
} 
Corollary 3.5. In the bluff equilibrium it holds that $q_{r}=F^{-1}\left(1-(1-c)^{r}\right), r \in \mathbb{N}$.

\section{Probability distribution of player actions}

In this section we evaluate the probability of saying yes and no by an active player under the bluff strategy. Knowing these probabilities enables us to derive recursive formulas for the expected number of rounds and the expected number of queries performed in the auction. Throughout this section, as well as in remaining sections, it is assumed that every player uses the bluff strategy.

Recall that in any round $r$ of the $c$-fraction auction, the query price $q_{r}$ is determined such that, conditional on $v_{i}$ belonging to $\left[p_{r}, u_{r}\right)$, the probability that $v_{i}$ is in $\left[p_{r}, q_{r}\right)$ is equal to $c$. We define $i_{r}=\min \left\{i \mid i \in A_{r}\right\}$-among the active players in round $r$ the one with the lowest ranking-and $j_{r}=\min \left\{i \mid i \in A_{r}, i \neq i_{r}\right\}$-among the active players in round $r$ the one with the second lowest ranking.

Proposition 4.1. In the bluff equilibrium it holds that a player $i \in A_{r}$ says yes in round $r$ with probability $1-c$, except when $i=i_{1}$ or $\left[i=j_{r}\right.$ and $i_{r}$ says no], in which case player $i$ says yes with probability 1.

Proof. First let us observe that when player $i_{r}$ says no for the first time, player $j_{r}$ says yes with certainty. Indeed, in all previous rounds player $i_{r}$ said yes and since $j_{r}$ is active in round $r$ also he said yes in those rounds. Both the payment and the query price have increased so that $p_{r}=q_{r-1}$. Since player $j_{r}$ follows the bluff strategy, his previous yes decision implies that $v_{j_{r}} \geqslant q_{r-1}=p_{r}$. If in round $r$ player $i_{r}$ says no, player $j_{r}$ is in the situation where he does not have an active predecessor with a yes decision and therefore says yes whenever his valuation is not smaller than $p_{r}$. It follows that after a round $r$ where player $i_{r}$ says no for the first time, he drops out, $j_{r}$ says yes and $i_{r+1}=j_{r}$. Otherwise, $i_{r+1}=i_{r}$.

Next we determine the probability that player $i_{r}$ says yes in round $r$. Having no active predecessor, player $i_{r}$ says yes if and only if $v_{i_{r}} \geqslant p_{r}$. Since $p_{1}=\alpha$ player $i_{1}$ says yes with certainty in round 1 . For $r>1$, we have that either $i_{r}=i_{r-1}$ or $i_{r}=j_{r-1}$. In both cases the decision of player $i_{r}$ in round $r-1$ was yes which happens if and only if $v_{i_{r}} \geqslant p_{r-1}$. Thus, the probability that $i_{r}$ says yes is equal to $\mathbb{P}\left(v_{i_{r}} \geqslant p_{r} \mid v_{i_{r}} \geqslant p_{r-1}\right)=\mathbb{P}\left(v_{i_{r}} \geqslant q_{r-1} \mid v_{i_{r}} \geqslant p_{r-1}\right)=1-c$. The last equality holds because $q_{r-1}$ satisfies

$\frac{F\left(q_{r-1}\right)-F\left(p_{r-1}\right)}{F\left(u_{r-1}\right)-F\left(p_{r-1}\right)}=c$,

and Theorem 3.4 implies that $u_{r-1}=\beta$.

Next we determine the probability of saying yes in round $r$ for any player $i \in A_{r} \backslash\left\{i_{r}\right\}$. We distinguish two cases.

First, consider the case where player $i_{r}$ says yes in round $r$. From the fact that $i \in A_{r}$, it follows that player $i$ said yes in round $r-1$ which is the case if and only if $v_{i} \geqslant q_{r-1}$. In round $r$ he says yes if and only if $v_{i} \geqslant q_{r}$. Thus, $\mathbb{P}\left(v_{i} \geqslant q_{r} \mid v_{i} \geqslant q_{r-1}\right)=\mathbb{P}\left(v_{i} \geqslant q_{r} \mid v_{i} \geqslant\right.$ $\left.p_{r}\right)=1-c$. The last equality holds for the same reason as above.

Secondly, consider the case where player $i_{r}$ says no in round $r$. As we have already shown, player $j_{r}$ says yes with certainty. For any player $i \in A_{r} \backslash\left\{i_{r}, j_{r}\right\}$, the situation is the same as in the previous case and thus such a player $i$ says yes with probability $1-c$.

Due to the query price setting rule of the $c$-fraction auction, Proposition 4.1 holds regardless of the distribution from which valuations are drawn. Moreover, the probability of saying yes or no by an active player does not depend on the round with the exception of round 1 . This enables us to derive recursive formulas for the expected number of rounds and the expected number of queries performed in the auction.

\section{Running time of the auction}

In this section we investigate the expected running time of the $c$-fraction auction if the bluff strategies are played. We analyze two measures, namely the expected number of rounds and the expected number of queries performed in the auction before the winner is found. As a query we consider each separate question of the auctioneer to an active player. For both measures, we first derive a recursive formula and then give an upper bound for the function defined by this formula.

\subsection{The expected number of rounds}

Let $e_{c}(k)$ be the expected number of rounds of the auction with $k$ active players, given that the decision of the active player with the lowest ranking is yes in the current round, and let $e_{c}^{*}(k)$ be the expected number of rounds given that this decision is no.

Consider round $r$ with $n$ active players and suppose that the decision of player $i_{r}$ in the current round is yes. The current round contributes 1 to $e_{c}(n)$. Now let us compute the expected number of remaining rounds. If all active players apart from player $i_{r}$ say no, the auction stops after this round. If $k$, where $1 \leqslant k \leqslant n-1, \mathrm{r}$ active players apart from player $i_{r}$ say yes, then the auction continues with $k+1$ active players. Using Proposition 4.1, the probability of this situation given the yes decision of player $i_{r}$ is $\left(\begin{array}{c}n-1 \\ k\end{array}\right)(1-c)^{k} c^{n-1-k}$. In the next round player $i_{r+1}=i_{r}$ says yes or no with probability $1-c$ and $c$ respectively. Thus if $k$ active players apart from player $i_{r}$ say yes in round $r$, the expected number of remaining rounds is equal to $(1-c) e_{c}(k+1)+c e_{c}^{*}(k+1)$. Hence, for any $n \geqslant 2$,

$$
e_{c}(n)=1+\sum_{k=1}^{n-1}\left(\begin{array}{c}
n-1 \\
k
\end{array}\right)(1-c)^{k} c^{n-1-k}\left[(1-c) e_{c}(k+1)+c e_{c}^{*}(k+1)\right] .
$$

Proposition 4.1 states that if player $i_{r+1}$ says no, player $j_{r+1}$ says yes with certainty, which causes player $i_{r+1}$ to drop out of the auction. For $k>1$ this yields $e_{c}^{*}(k+1)=e_{c}(k)$, as the expected number of rounds with player $i_{r+1}$ saying no is equal to the expected number of rounds without this player and the player with lowest rank among the remaining $k$ players saying no. Observe that $e_{c}^{*}(2)=1$ as exactly one of the two players says yes.

We denote $P_{k}^{n}=\left(\begin{array}{l}n \\ k\end{array}\right)(1-c)^{k} c^{n-k}$ and rewrite Eq. (1) as follows:

$$
\begin{aligned}
e_{c}(n)= & 1+\sum_{k=1}^{n-1} P_{k}^{n-1}\left[(1-c) e_{c}(k+1)+c e_{c}^{*}(k+1)\right] \\
= & 1+(1-c) \sum_{k=1}^{n-2} P_{k}^{n-1} e_{c}(k+1)+(1-c) P_{n-1}^{n-1} e_{c}(n) \\
& +c \sum_{k=2}^{n-1} P_{k}^{n-1} e_{c}^{*}(k+1)+c P_{1}^{n-1} e_{c}^{*}(2) \\
= & 1+(1-c) \sum_{k=1}^{n-2} P_{k}^{n-1} e_{c}(k+1)+(1-c)^{n} e_{c}(n) \\
& +c \sum_{k=2}^{n-1} P_{k}^{n-1} e_{c}(k)+(n-1)(1-c) c^{n-1} \\
= & 1+(1-c)^{n} e_{c}(n)+(n-1)(1-c) c^{n-1} \\
& +(1-c) \sum_{k=2}^{n-1} P_{k-1}^{n-1} e_{c}(k)+c \sum_{k=2}^{n-1} P_{k}^{n-1} e_{c}(k) \\
= & 1+(1-c)^{n} e_{c}(n)+(n-1)(1-c) c^{n-1} \\
& +\sum_{k=2}^{n-1}\left[(1-c) P_{k-1}^{n-1}+c P_{k}^{n-1}\right] e_{c}(k) \\
= & 1+(1-c)^{n} e_{c}(n)+(n-1)(1-c) c^{n-1}+\sum_{k=2}^{n-1} P_{k}^{n} e_{c}(k)
\end{aligned}
$$


Table 1

The expected number of rounds $e_{c}(n)$ in the $c$-fraction auction.

\begin{tabular}{|c|c|c|c|c|c|c|c|c|c|c|c|}
\hline \multirow[t]{2}{*}{$n$} & \multicolumn{11}{|l|}{$c$} \\
\hline & $1 / 10$ & $1 / 8$ & $1 / 6$ & $1 / 4$ & $1 / 3$ & $1 / 2$ & $2 / 3$ & $3 / 4$ & $5 / 6$ & $7 / 8$ & $9 / 10$ \\
\hline 2 & 5.737 & 4.733 & 3.727 & 2.714 & 2.200 & 1.667 & 1.375 & 1.267 & 1.171 & 1.127 & 1.101 \\
\hline 3 & 8.901 & 7.230 & 5.555 & 3.873 & 3.021 & 2.143 & 1.663 & 1.483 & 1.319 & 1.240 & 1.193 \\
\hline 4 & 11.273 & 9.102 & 6.927 & 4.742 & 3.638 & 2.505 & 1.891 & 1.660 & 1.446 & 1.341 & 1.277 \\
\hline 5 & 13.172 & 10.600 & 8.024 & 5.437 & 4.131 & 2.794 & 2.076 & 1.807 & 1.557 & 1.431 & 1.353 \\
\hline 6 & 14.753 & 11.848 & 8.938 & 6.016 & 4.542 & 3.035 & 2.230 & 1.931 & 1.654 & 1.512 & 1.423 \\
\hline 7 & 16.109 & 12.918 & 9.721 & 6.513 & 4.895 & 3.241 & 2.361 & 2.037 & 1.738 & 1.584 & 1.486 \\
\hline 8 & 17.296 & 13.854 & 10.407 & 6.947 & 5.203 & 3.421 & 2.475 & 2.129 & 1.813 & 1.650 & 1.545 \\
\hline 9 & 18.350 & 14.686 & 11.016 & 7.334 & 5.477 & 3.581 & 2.576 & 2.211 & 1.879 & 1.709 & 1.598 \\
\hline 10 & 19.299 & 15.435 & 11.565 & 7.681 & 5.724 & 3.726 & 2.667 & 2.283 & 1.939 & 1.762 & 1.647 \\
\hline 20 & 25.647 & 20.443 & 15.233 & 10.006 & 7.373 & 4.690 & 3.275 & 2.760 & 2.312 & 2.102 & 1.971 \\
\hline 30 & 29.417 & 23.418 & 17.412 & 11.387 & 8.353 & 5.264 & 3.637 & 3.048 & 2.524 & 2.281 & 2.140 \\
\hline 40 & 32.109 & 25.541 & 18.967 & 12.372 & 9.052 & 5.673 & 3.894 & 3.255 & 2.681 & 2.406 & 2.249 \\
\hline 50 & 34.203 & 27.194 & 20.177 & 13.140 & 9.596 & 5.991 & 4.095 & 3.414 & 2.808 & 2.508 & 2.333 \\
\hline 60 & 35.918 & 28.547 & 21.168 & 13.768 & 10.042 & 6.252 & 4.260 & 3.543 & 2.913 & 2.595 & 2.405 \\
\hline 70 & 37.370 & 29.693 & 22.007 & 14.299 & 10.419 & 6.472 & 4.399 & 3.652 & 3.002 & 2.672 & 2.469 \\
\hline 80 & 38.628 & 30.686 & 22.735 & 14.760 & 10.746 & 6.664 & 4.520 & 3.747 & 3.077 & 2.740 & 2.527 \\
\hline 90 & 39.740 & 31.563 & 23.377 & 15.167 & 11.035 & 6.833 & 4.627 & 3.831 & 3.143 & 2.801 & 2.580 \\
\hline 100 & 40.735 & 32.348 & 23.952 & 15.532 & 11.294 & 6.984 & 4.722 & 3.907 & 3.201 & 2.855 & 2.629 \\
\hline
\end{tabular}

This can be rewritten to

$$
\begin{aligned}
{\left[1-(1-c)^{n}\right] e_{c}(n)=} & 1+(n-1)(1-c) c^{n-1} \\
& +\sum_{k=2}^{n-1}\left(\begin{array}{l}
n \\
k
\end{array}\right)(1-c)^{k} c^{n-k} e_{c}(k) .
\end{aligned}
$$

This formula is valid for any $n \geqslant 2$.

Now notice that since in the first round player $i_{1}$ says yes with certainty, the expected number of rounds of the auction with $n$ players is equal to $e_{c}(n)$. Thus, using Formula 2, we can compute the expected number of rounds in the auction of $n$ players. Plugging in $n=2$ yields $e_{c}(2)=\frac{1+c(1-c)}{c(2-c)}$. All other values can be determined recursively. Table 1 presents the computational results for different values of $c$ in the auction with up to 100 players. Notice that the bisection character of the $c$-fraction auction guarantees a remarkably low number of rounds. For instance, with 100 bidders and $c=1 / 2$, the auction terminates in less than 7 rounds on average.

Fig. 1(a) shows how for a fixed value of $c$ the expected number of rounds increases in the number of players who participate in the auction. Furthermore, Fig. 1(b) demonstrates how for a fixed number of players the expected number of rounds decreases as $c$ increases.

We show next that the expected number of rounds of the auction is bounded from above by a function that is logarithmic in the number of players. We determine this bound for $c \leqslant 1 / 2$, since the

(a)

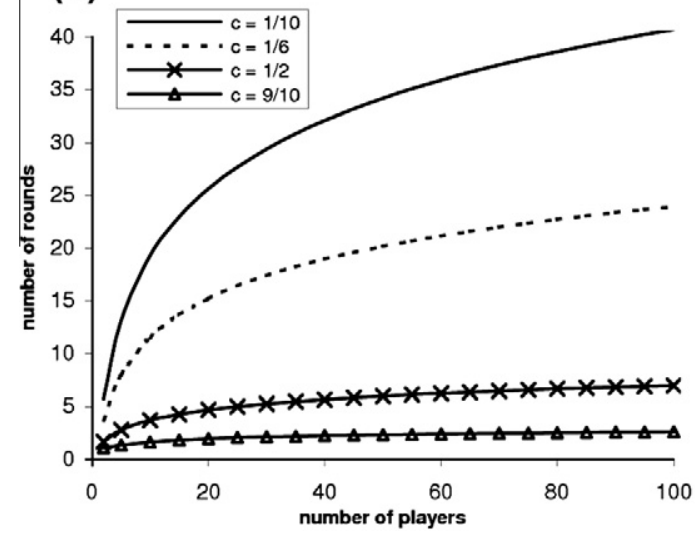

bound for $\bar{c}=1 / 2$ is also valid for $c>1 / 2$. First, we introduce some notation and several lemmas.

For any $n \geqslant 2$, define $D_{n}=\prod_{k=1}^{n} \frac{1}{1-(1-c)^{k}}$. Also, define $E_{2}=\frac{1+c(1-c)}{c(2-c)}$ and, for any $n>2$,

$E_{n}=1+(n-1)(1-c) c^{n-1}+\sum_{k=2}^{n-1}\left(\begin{array}{l}n \\ k\end{array}\right)(1-c)^{k} c^{n-k} E_{k}$.

Lemma 5.1. For any $n \geqslant 2, e_{c}(n)<E_{n} \cdot D_{n}$.

Proof. The proof is by induction on $n$. The basis of the induction is trivial since $e_{c}(2)=E_{2}$ and $D_{2}>1$. Suppose that $e_{c}(k)<E_{k} \cdot D_{k}$ is true for any $2 \leqslant k \leqslant n-1$. Notice that $D_{n}>D_{n-1}>\cdots>D_{2}>1$. Thus, using the recursive formula for $e_{c}(n)$ and the induction hypothesis,

$$
\begin{aligned}
{\left[1-(1-c)^{n}\right] e_{c}(n)=} & 1+(n-1)(1-c) c^{n-1}+\sum_{k=2}^{n-1}\left(\begin{array}{l}
n \\
k
\end{array}\right)(1-c)^{k} c^{n-k} e_{c}(k) \\
& <1+(n-1)(1-c) c^{n-1}+\sum_{k=2}^{n-1}\left(\begin{array}{l}
n \\
k
\end{array}\right)(1-c)^{k} c^{n-k} E_{k} D_{k} \\
& <1+(n-1)(1-c) c^{n-1}+\sum_{k=2}^{n-1}\left(\begin{array}{l}
n \\
k
\end{array}\right)(1-c)^{k} c^{n-k} E_{k} D_{n-1} \\
& <D_{n-1}\left[1+(n-1)(1-c) c^{n-1}+\sum_{k=2}^{n-1}\left(\begin{array}{l}
n \\
k
\end{array}\right)(1-c)^{k} c^{n-k} E_{k}\right] \\
& =E_{n} \cdot D_{n-1},
\end{aligned}
$$

(b)

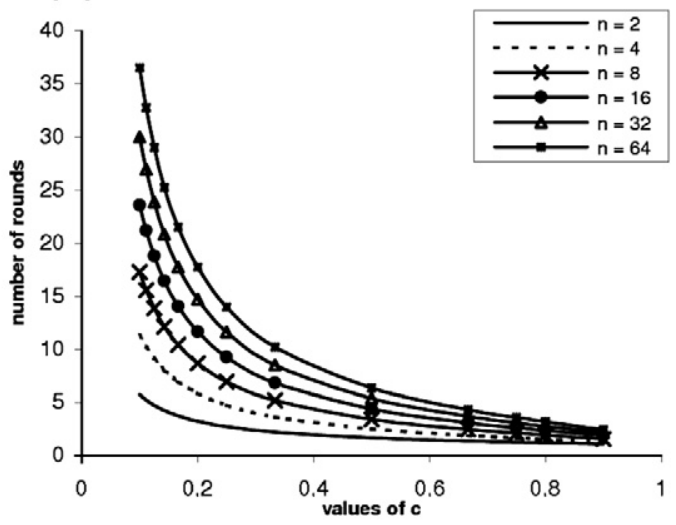

Fig. 1. The expected number of rounds (a) for different fixed values of $c$; (b) for different fixed numbers of players. 


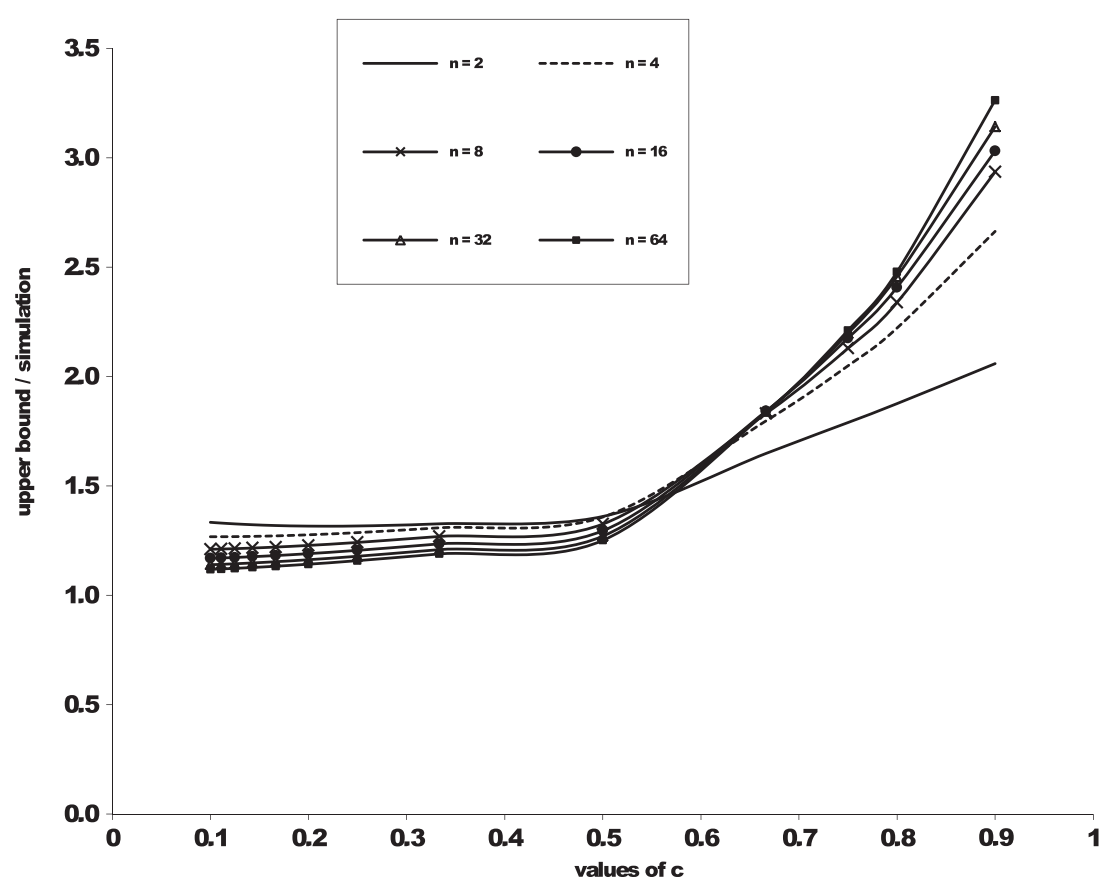

Fig. 2. The ratio between the upper bound from Theorem 5.4 and the results from Table 1 .

which completes the proof.

Now we find bounds on $D_{n}$ and $E_{n}$.

Lemma 5.2. For any $n \geqslant 2, D_{n}<e^{\frac{1-c}{c^{2}}}$.

Proof. We have to show that $\ln D_{n}<\frac{1-c}{c^{2}}$. Let us define $\lambda=\frac{1}{1-c}$. Notice that since $0<c<1$ it holds that $\lambda>1$.

We have

$$
\begin{aligned}
\ln D_{n} & =\ln \left(\prod_{k=1}^{n} \frac{\lambda^{k}}{\lambda^{k}-1}\right)=\sum_{k=1}^{n}\left[\ln \lambda^{k}-\ln \left(\lambda^{k}-1\right)\right] \leqslant \sum_{k=1}^{n} \partial_{x} \ln x_{\mid x=\lambda^{k}-1} \\
& =\sum_{k=1}^{n} \frac{1}{\lambda^{k}-1}<\sum_{k=1}^{n} \frac{1}{\lambda^{k}-\lambda^{k-1}}=\frac{1}{\lambda-1} \sum_{k=1}^{n} \frac{1}{\lambda^{k-1}}<\frac{1}{\lambda-1} \sum_{k=0}^{\infty} \frac{1}{\lambda^{k}} \\
& =\frac{\lambda}{(\lambda-1)^{2}}=\frac{1-c}{c^{2}},
\end{aligned}
$$

where the first inequality holds because of the concavity of $\ln$.

Lemma 5.3. For any $n \geqslant 2$ and any $c \leqslant \frac{1}{2}, E_{n}<1+\log _{a} n$, with base $a=\frac{1}{1-c^{*}}$

Proof. The proof is by induction on $n$. The basis of the induction holds since $\frac{1+c(1-c)}{c(2-c)}<1+\log _{a} 2$ for any $c \leqslant \frac{1}{2}$. Suppose $E_{k}<1+\log _{a} k$ for any $2 \leqslant k \leqslant n-1$. Using the induction hypothesis,

$$
\begin{aligned}
E_{n}= & +(n-1)(1-c) c^{n-1}+\sum_{k=2}^{n-1}\left(\begin{array}{l}
n \\
k
\end{array}\right)(1-c)^{k} c^{n-k} E_{k} \\
& <1+(n-1)(1-c) c^{n-1}+\sum_{k=2}^{n-1}\left(\begin{array}{l}
n \\
k
\end{array}\right)(1-c)^{k} c^{n-k}\left(\log _{a} k+1\right) \\
& <1+\sum_{k=2}^{n-1}\left(\begin{array}{l}
n \\
k
\end{array}\right)(1-c)^{k} c^{n-k} \log _{a} k+\sum_{k=1}^{n-1}\left(\begin{array}{l}
n \\
k
\end{array}\right)(1-c)^{k} c^{n-k} \\
& <2+\sum_{k=2}^{n-1}\left(\begin{array}{l}
n \\
k
\end{array}\right)(1-c)^{k} c^{n-k} \log _{a} k .
\end{aligned}
$$

Since the logarithm with base $a=\frac{1}{1-c}$ is concave, we know that if $\lambda_{k} \geqslant 0$ and $\sum_{k=0}^{n} \lambda_{k}=1$, then

$$
\sum_{k=0}^{n} \lambda_{k} \log _{a}\left(x_{k}\right) \leqslant \log _{a}\left(\sum_{k=0}^{n} \lambda_{k} x_{k}\right) .
$$

So let us take $\lambda_{k}=\left(\begin{array}{l}n \\ k\end{array}\right)(1-c)^{k} c^{n-k}$ for $k=0, \ldots, n$ and take $x_{0}=x_{n}=1, x_{k}=k$ for any $1 \leqslant k \leqslant n-1$.

Then

$$
\begin{aligned}
& E_{n}<2+\sum_{k=2}^{n-1}\left(\begin{array}{l}
n \\
k
\end{array}\right)(1-c)^{k} c^{n-k} \log _{a} k \\
& =2+\sum_{k=0}^{n}\left(\begin{array}{l}
n \\
k
\end{array}\right)(1-c)^{k} c^{n-k} \log _{a}\left(x_{k}\right) \\
& \leqslant 2+\log _{a}\left[\sum_{k=0}^{n}\left(\begin{array}{l}
n \\
k
\end{array}\right)(1-c)^{k} c^{n-k} x_{k}\right] \\
& =2+\log _{a}\left[\sum_{k=1}^{n-1}\left(\begin{array}{l}
n \\
k
\end{array}\right)(1-c)^{k} c^{n-k} k+c^{n}+(1-c)^{n}\right] \\
& \leqslant 2+\log _{a}\left[\sum_{k=1}^{n-1}\left(\begin{array}{l}
n \\
k
\end{array}\right)(1-c)^{k} c^{n-k} k+n(1-c)^{n}\right] \\
& =2+\log _{a}\left[\sum_{k=0}^{n}\left(\begin{array}{l}
n \\
k
\end{array}\right)(1-c)^{k} c^{n-k} k\right] \\
& =2+\log _{a}[(1-c) n]=1+\log _{a} n .
\end{aligned}
$$

The last inequality holds since for any $c \leqslant \frac{1}{2}$ and any $n \geqslant 2$ it holds that $c^{n}+(1-c)^{n} \leqslant 2(1-c)^{n} \leqslant n(1-c)^{n}$.

A final immediate consequence of Lemmas $5.1-5.3$ is the following theorem.

Theorem 5.4. For any $c \leqslant \frac{1}{2}$ and any $n \geqslant 2, e_{c}(n) \leqslant e^{\frac{1-c}{c^{2}}}\left(\log _{\frac{1}{1-}} n+1\right)$.

Since $e_{c}(n)<e_{\bar{c}}(n)$ when $c>\bar{c}$, the upper bound for $\bar{c}=\frac{1}{2}$ is also valid for any $c>\frac{1}{2}$.

We have shown that the expected number of rounds of the $c$ fraction auction is bounded from above by a function that is logarithmic in the number of players. Furthermore, a comparison of the bound with the computed results shows that for a fixed value of $c$ the ratio between the bound and the computed result is approximately constant as a function of $n$, implying that the bound has approximately the correct order of magnitude. Fig. 2 shows the 
Table 2

The expected number of queries $b_{c}(n)$ in the $c$-fraction auction.

\begin{tabular}{|c|c|c|c|c|c|c|c|c|c|c|c|}
\hline \multirow[t]{2}{*}{$n$} & \multicolumn{11}{|l|}{$c$} \\
\hline & $1 / 10$ & $1 / 8$ & $1 / 6$ & $1 / 4$ & $1 / 3$ & $1 / 2$ & $2 / 3$ & $3 / 4$ & $5 / 6$ & $7 / 8$ & $9 / 10$ \\
\hline 2 & 11.474 & 9.467 & 7.455 & 5.429 & 4.400 & 3.333 & 2.750 & 2.533 & 2.343 & 2.254 & 2.202 \\
\hline 3 & 21.790 & 17.779 & 13.759 & 9.718 & 7.674 & 5.571 & 4.442 & 4.029 & 3.666 & 3.496 & 3.396 \\
\hline 4 & 32.027 & 26.013 & 19.988 & 13.935 & 10.879 & 7.752 & 6.094 & 5.495 & 4.972 & 4.727 & 4.582 \\
\hline 5 & 42.217 & 34.200 & 26.171 & 18.109 & 14.044 & 9.897 & 7.717 & 6.938 & 6.264 & 5.949 & 5.762 \\
\hline 6 & 52.375 & 42.356 & 32.323 & 22.254 & 17.181 & 12.017 & 9.320 & 8.365 & 7.545 & 7.162 & 6.936 \\
\hline 7 & 62.511 & 50.490 & 38.454 & 26.378 & 20.298 & 14.120 & 10.908 & 9.778 & 8.815 & 8.368 & 8.104 \\
\hline 8 & 72.630 & 58.607 & 44.568 & 30.487 & 23.401 & 16.211 & 12.484 & 11.180 & 10.078 & 9.568 & 9.268 \\
\hline 9 & 82.735 & 66.711 & 50.669 & 34.583 & 26.492 & 18.291 & 14.051 & 12.575 & 11.333 & 10.763 & 10.427 \\
\hline 10 & 92.830 & 74.804 & 56.761 & 38.670 & 29.575 & 20.363 & 15.611 & 13.962 & 12.582 & 11.952 & 11.582 \\
\hline 20 & 193.465 & 155.430 & 117.372 & 79.251 & 60.124 & 40.845 & 31.016 & 27.653 & 24.893 & 23.679 & 22.985 \\
\hline 30 & 293.842 & 235.802 & 177.735 & 119.597 & 90.451 & 61.132 & 46.258 & 41.203 & 37.070 & 35.264 & 34.248 \\
\hline 40 & 394.111 & 316.068 & 237.995 & 159.843 & 120.684 & 81.336 & 61.430 & 54.691 & 49.201 & 46.802 & 45.457 \\
\hline 50 & 494.320 & 396.274 & 298.196 & 200.035 & 150.865 & 101.495 & 76.563 & 68.144 & 61.307 & 58.319 & 56.644 \\
\hline 60 & 594.492 & 476.443 & 358.361 & 240.192 & 181.014 & 121.626 & 91.673 & 81.574 & 73.394 & 69.825 & 67.820 \\
\hline 70 & 694.637 & 556.587 & 418.501 & 280.325 & 211.140 & 141.736 & 106.766 & 94.989 & 85.468 & 81.320 & 78.989 \\
\hline 80 & 794.763 & 636.711 & 478.622 & 320.440 & 241.249 & 161.832 & 121.847 & 108.394 & 97.531 & 92.809 & 90.152 \\
\hline 90 & 894.874 & 716.820 & 538.729 & 360.542 & 271.345 & 181.916 & 136.918 & 121.790 & 109.586 & 104.290 & 101.311 \\
\hline 100 & 994.973 & 796.918 & 598.825 & 400.633 & 301.431 & 201.992 & 151.981 & 135.180 & 121.634 & 115.766 & 112.466 \\
\hline
\end{tabular}

ratio between the upper bound from Theorem 5.4 and the results from Table 1 for up to 64 bidders and different values for $c$. The ratio increases for large $c>\frac{1}{2}$, as we use the bound for $c=\frac{1}{2}$, but is almost constant for $c \leqslant \frac{1}{2}$, independent of $n$.

\subsection{The expected number of queries}

Let $b_{c}(k)$ be the expected number of queries of the auction with $k$ active players, given that the decision of the active player with the lowest ranking is yes in the current round, and let $b_{c}^{*}(k)$ be the expected number of queries given that this decision is no. Notice that in a round with $k$ active players, $k$ queries are performed. Following the same argument as we used for determining the formula for the expected number of rounds, we find that for any $n \geqslant 2$

$b_{c}(n)=n+\sum_{k=1}^{n-1}\left(\begin{array}{c}n-1 \\ k\end{array}\right)(1-c)^{k} c^{n-1-k}\left[(1-c) b_{c}(k+1)+c b_{c}^{*}(k+1)\right]$.

Again, notice that when player $i_{r+1}=i_{r}$ says no, $\mathrm{r}$ player $j_{r+1}$ says yes with certainty, which causes player $i_{r+1}$ to drop out of the auction. Thus, $b_{c}^{*}(2)=2$ and for all $k \geqslant 2$ it holds that $b_{c}^{*}(k+1)=1+b_{c}(k)$.

Recall that $P_{k}^{n}=\left(\begin{array}{l}n \\ k\end{array}\right)(1-c)^{k} c^{n-k}$. Using the facts that $b^{*}(2)=2$ and $b^{*}(k+1)=b(k)+1$ for all $k \geqslant 2$, we get from (3) that

$$
\begin{aligned}
b_{c}(n)= & n+\sum_{k=1}^{n-1} P_{k}^{n-1}\left[(1-c) b_{c}(k+1)+c b_{c}^{*}(k+1)\right]=n+(1-c) \\
& \times \sum_{k=1}^{n-2} P_{k}^{n-1} b_{c}(k+1)+(1-c) P_{n-1}^{n-1} b_{c}(n)+c \sum_{k=2}^{n-1} P_{k}^{n-1} b_{c}^{*}(k+1) \\
& +c P_{1}^{n-1} b_{c}^{*}(2)=n+(1-c) \sum_{k=1}^{n-2} P_{k}^{n-1} b_{c}(k+1)+(1-c)^{n} b_{c}(n) \\
& +c \sum_{k=2}^{n-1} P_{k}^{n-1}\left[b_{c}(k)+1\right]+2(n-1)(1-c) c^{n-1}=n+(1-c)^{n} b_{c}(n) \\
& +2(n-1)(1-c) c^{n-1}+(1-c) \sum_{k=2}^{n-1} P_{k-1}^{n-1} b_{c}(k)+c \sum_{k=2}^{n-1} P_{k}^{n-1} b_{c}(k) \\
& +c \sum_{k=2}^{n-1} P_{k}^{n-1}=n+(1-c)^{n} b_{c}(n)+2(n-1)(1-c) c^{n-1} \\
& +c-c^{n}-(n-1)(1-c) c^{n-1}+\sum_{k=2}^{n-1}\left[(1-c) P_{k-1}^{n-1}+c P_{k}^{n-1}\right] b_{c}(k) \\
= & n+(1-c)^{n} b_{c}(n)+(n-1)(1-c) c^{n-1}+c-c^{n}+\sum_{k=2}^{n-1} P_{k}^{n} b_{c}(k) .
\end{aligned}
$$

Rewriting yields, for any $n \geqslant 2$,

$$
\begin{aligned}
{\left[1-(1-c)^{n}\right] b_{c}(n)=} & n+(n-1)(1-c) c^{n-1}+c-c^{n} \\
& +\sum_{k=2}^{n-1}\left(\begin{array}{l}
n \\
k
\end{array}\right)(1-c)^{k} c^{n-k} b_{c}(k) .
\end{aligned}
$$

Now notice that since in the first round player $i_{1}$ says yes with certainty, the expected number of queries in the auction of $n$ players is equal to $b_{c}(n)$. Thus using (4) we can compute the expected number of queries performed in the auction with $n$ players. Substituting in $n=2$ yields $b_{c}(2)=\frac{2+2 c(1-c)}{c(2-c)}$. All other values can be determined recursively. Table 2 presents the computational results for different values of $c$ in the auction with up to 100 players. Again, the $c$-fraction auction needs only very few queries to allocate the object. For instance, with 100 bidders and $c=1 / 2$, the auction needs less than 202 queries on average, surprisingly little if one realizes that 100 is the absolute minimum with 100 bidders.

Fig. 3(a) demonstrates that for a fixed value of $c$, the expected number of queries increases in the number of players participating in the auction. Fig. 3(b) shows that for a fixed number of players, the expected number of queries decreases as $c$ becomes larger.

We show next that the expected number of queries is bounded from above by a function that is linear in the number of players. To prove this we introduce some notation and several lemmas.

Define $B_{2}=\frac{2+2 c(1-c)}{c(2-c)}$ and, for any $n>2$,

$B_{n}=n+(n-1)(1-c) c^{n-1}+c-c^{n}+\sum_{k=2}^{n-1}\left(\begin{array}{l}n \\ k\end{array}\right)(1-c)^{k} c^{n-k} B_{k}$.

Recall that $D_{n}=\prod_{k=1}^{n} \frac{1}{1-(1-c)^{k}}$.

Lemma 5.5. For any $n \geqslant 2, b_{c}(n) \leqslant B_{n} \cdot D_{n}$.

Proof. The proof is identical to the proof of Lemma 5.1 if we replace $e_{c}(k)$ by $b_{c}(k)$ and $E_{k}$ by $B_{k}$ for all $2 \leqslant k \leqslant n$.

From Lemma 5.2 we know that for any $n \geqslant 2, D_{n} \leqslant e^{\frac{1-c}{c^{2}}}$. We find now a bound on $B_{n}$.

Lemma 5.6. For any $n \geqslant 2, B_{n} \leqslant\left(\frac{2}{c}+\frac{1}{2}\right)(n+1)$.

Proof. The proof is by induction on $n$. The basis of the induction holds since it can be easily shown that $B_{2}<3\left(\frac{2}{c}+\frac{1}{2}\right)$. Now suppose that $B_{k} \leqslant\left(\frac{2}{c}+\frac{1}{2}\right)(k+1)$ for any $2 \leqslant k \leqslant n-1$. Using the induction hypothesis, 
(a)

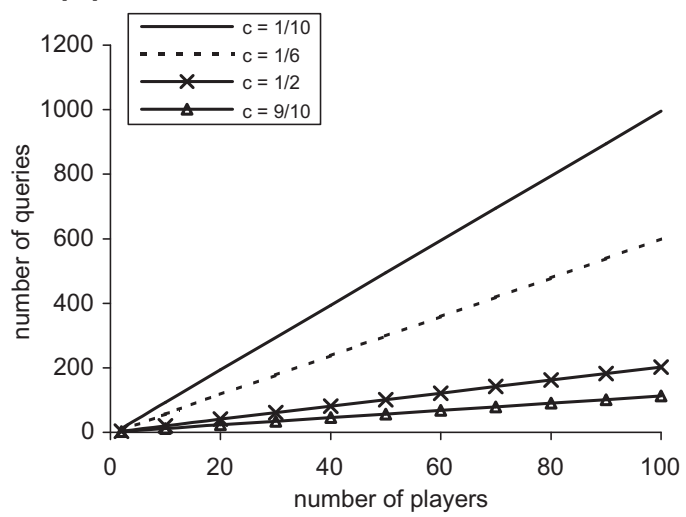

(b)

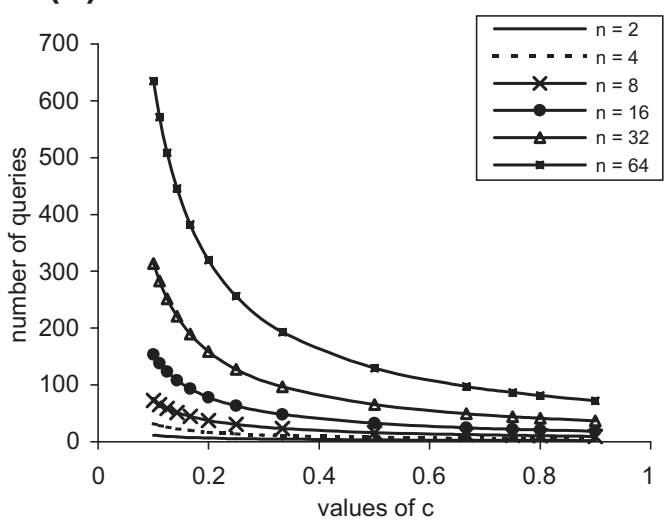

Fig. 3. The expected number of queries (a) for different fixed values of $c$; (b) for different fixed numbers of players.

$$
\begin{aligned}
B_{n}= & n+(n-1)(1-c) c^{n-1}+c-c^{n}+\sum_{k=2}^{n-1}\left(\begin{array}{l}
n \\
k
\end{array}\right)(1-c)^{k} c^{n-k} B_{k} \\
& \leqslant n+(n-1)(1-c) c^{n-1}+c-c^{n}+\sum_{k=2}^{n-1}(k)(1-c)^{k} c^{n-k}\left(\frac{2}{c}+\frac{1}{2}\right) \\
& \times(k+1) \leqslant 2 n+c+\left(\frac{2}{c}+\frac{1}{2}\right) \sum_{k=0}^{n}\left(\begin{array}{l}
n \\
k
\end{array}\right)(1-c)^{k} c^{n-k} k+\left(\frac{2}{c}+\frac{1}{2}\right) \\
& \times \sum_{k=0}^{n}\left(\begin{array}{l}
n \\
k
\end{array}\right)(1-c)^{k} c^{n-k}=2 n+c+\left(\frac{2}{c}+\frac{1}{2}\right)(1-c) n \\
& +\left(\frac{2}{c}+\frac{1}{2}\right)=\left(\frac{2}{c}+\frac{1}{2}\right)(n+1)+c\left(1-\frac{n}{2}\right) \leqslant\left(\frac{2}{c}+\frac{1}{2}\right)(n+1) .
\end{aligned}
$$

The last inequality holds since $n \geqslant 2$.

A final immediate consequence of Lemmas 5.2, 5.5, 5.6 is the following theorem.

Theorem 5.7. For any integer $n \geqslant 2, b_{c}(n) \leqslant e^{\frac{1-c}{c^{2}}}\left(\frac{2}{c}+\frac{1}{2}\right)(n+1)$.

We have shown that the expected number of queries is bounded from above by a function that is linear in the number of players. Again, a comparison of the bound with the computed results suggests that this bound is not tight. It can be easily checked that for a fixed value of $c$ the ratio between the bound and the computed result is approximately constant as a function of $n$, implying that the bound is likely to have the correct order of magnitude.

\section{Efficiency of the auction}

In this section we investigate the efficiency of the $c$-fraction auction when the bluff equilibrium is played. In particular, in the following two subsections, we compute the probability of inefficient allocation and the expected loss of welfare.

\subsection{The probability of inefficient allocation}

We derive a recursive formula for the probability of inefficient allocation and give an upper bound for this probability using the recursive formula. We denote the probability that the auction with $n$ players terminates in an inefficient allocation by $P_{c}(n)$.

A first important observation is that the $c$-fraction auction with $k$ players having valuations drawn from $F$ conditional on these valuations being greater than or equal to $F^{-1}(c)$ has exactly the same structure as the original $c$-fraction auction with $k$ players having valuations drawn from $F$. For both cases, the probability of an inefficient allocation under the bluff equilibrium is the same.

Consider the case where the valuation of all players is smaller than $F^{-1}(c)$. The probability of this event is $c^{n}$. In this case player $i_{1}$ is the only player saying yes, rand therefore receives the object. The auction is only efficient if the player with the lowest ranking has the highest valuation, which happens with probability $1 / n$. Thus this case contributes $((n-1) / n) c^{n}$ to $P_{c}(n)$. Next consider the case where $k \geqslant 1$ players have valuations larger than or equal to $F^{-1}(c)$ and $n-k$ players have valuations smaller than $F^{-1}(c)$, which happens with probability $\left(\begin{array}{l}n \\ k\end{array}\right) c^{n-k}(1-c)^{k}$. For $k=1$ the auction is efficient, so this case adds zero to $P_{c}(n)$. Consider the case where $k>1$. Either player $i_{1}$ has a value below $F^{-1}(c)$, responds no in round 2 , and inefficiency among the remaining $k$ bidders takes place with probability $P_{c}(k)$. Or player $i_{1}$ has a value greater than or equal to $F^{-1}(c)$, in which case the auction starts in round 2 with $k$ bidders having a value greater than or equal to $F^{-1}(c)$ and inefficiency takes place with probability $P_{c}(k)$. We find that

$P_{c}(n)=\frac{n-1}{n} c^{n}+\sum_{k=2}^{n}\left(\begin{array}{l}n \\ k\end{array}\right) c^{n-k}(1-c)^{k} P_{c}(k)$.

A direct evaluation of the recursive formula yields that $P_{c}(2)=\frac{1}{2} \cdot \frac{c}{2-c}$. For $n>2$, rewriting leads to

$\left[1-(1-c)^{n}\right] P_{c}(n)=\frac{n-1}{n} c^{n}+\sum_{k=2}^{n-1}\left(\begin{array}{l}n \\ k\end{array}\right) c^{n-k}(1-c)^{k} P_{c}(k)$.

A direct computation of this expression for different combinations of $n$ and $c$ gives the values that are plotted in Fig. 4 .

The recursive formula can also be used to derive the following upper bound on $P_{c}(n)$.

Theorem 6.1. For all $n \in \mathbb{N}, P_{c}(n)<c$.

Proof. The proof is by induction on $n$. The basis of the induction holds since $P_{c}(1)=0$ and $P_{c}(2)=\frac{1}{2} \cdot \frac{c}{2-c}<c$. Suppose that $n \geqslant 3$ and $P_{c}(k)<c$ for all $1 \leqslant k \leqslant n-1$. Then

$$
\begin{aligned}
P_{c}(n) & =\frac{1}{1-(1-c)^{n}}\left[\frac{n-1}{n} \cdot c^{n}+\sum_{k=2}^{n-1}\left(\begin{array}{l}
n \\
k
\end{array}\right) c^{n-k}(1-c)^{k} \cdot P_{c}(k)\right] \\
& <\frac{1}{1-(1-c)^{n}}\left[c^{n}+c \sum_{k=2}^{n-1}\left(\begin{array}{l}
n \\
k
\end{array}\right) c^{n-k}(1-c)^{k}\right] \\
& =\frac{1}{1-(1-c)^{n}}\left[c^{n}+c\left(1-c^{n}-n(1-c) c^{n-1}-(1-c)^{n}\right)\right] \\
& =\frac{c\left(1-(1-c)^{n}\right)}{1-(1-c)^{n}}+\frac{c^{n}-c^{n+1}-n(1-c) c^{n}}{1-(1-c)^{n}} \\
& =c+\frac{c^{n}(1-c)(1-n)}{1-(1-c)^{n}}<c .
\end{aligned}
$$




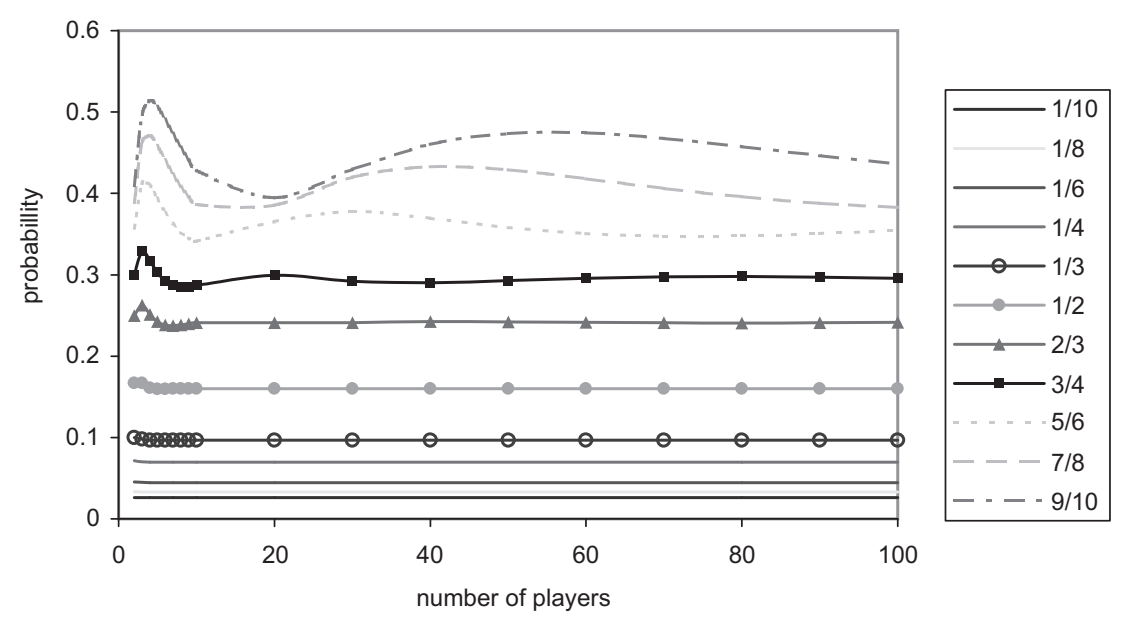

Fig. 4. The probability of inefficient allocation.

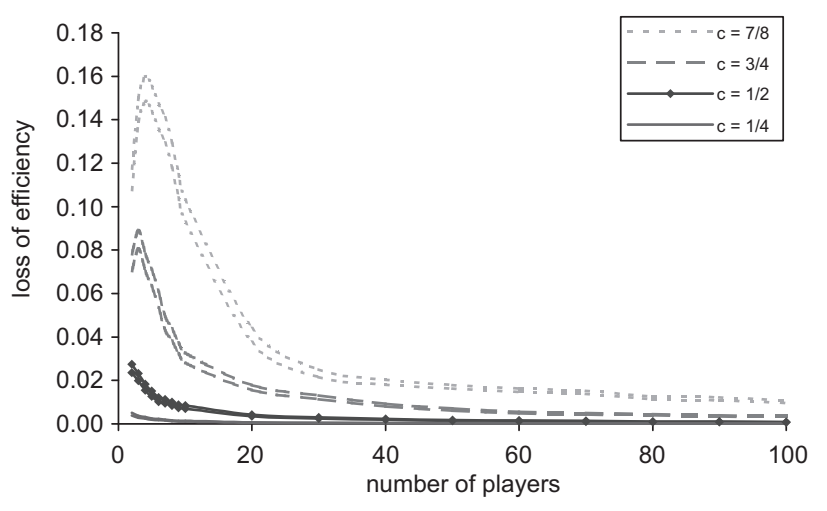

Fig. 5. The expected loss of welfare, $99 \%$ confidence interval.

The first inequality holds by the induction assumption and the fact that $\frac{n-1}{n}<1$. The last inequality holds since $n \geqslant 3$.

This theorem shows in particular that by choosing an appropriate fraction $c$ in the auction we can make the probability of inefficiency as small as we like, independent of the number of players! Also Fig. 4 shows that the probability of inefficient allocation is quite independent from the number of players.

When we assume $c<\frac{1}{2}$, it can be shown by the same chain of arguments that $P_{c}(n) \leqslant \frac{1}{2} c$ for all $n \in \mathbb{N}$.

\subsection{The expected loss of welfare}

The welfare of an auction is equal to the valuation of the winner of the auction. Given $v$, the maximum welfare is $\max \left\{v_{i} \mid i \in N\right\}$. The expected loss of welfare, denoted by $L_{c}(n)$, is the expected value of the difference between the maximum welfare and the valuation of the winner. To estimate the value of $L_{c}(n)$, we simulated the $c$-fraction auction and ran it for valuations uniformly and independently drawn from the interval $[0,1)$. For each combination of the value $c$ and the number of players $n$, we ran 10,000 trials. Fig. 5 shows the $99 \%$ confidence interval for the expected loss of welfare. It is interesting to notice that the maximum expected loss does not occur when the number of players is minimal.

For a distribution function $F$, we define $\gamma(c) \in \mathbb{R}_{+}$by

$\gamma(c)=\sup _{r \in \mathbb{N}}\left\{F^{-1}\left(1-(1-c)^{r}\right)-F^{-1}\left(1-(1-c)^{r-1}\right)\right\}$,

so $\gamma(c)$ measures the maximal difference between the query price $q_{r}$ and the payment $p_{r}$ that can occur in an auction. We restrict ourselves to distributions for which $\gamma(c)$ is finite. It is easily verified that for the uniform distribution $\gamma(c)$ is equal to $c$ and for the exponential distribution with parameter $\lambda$ we have $\gamma(c)=-(\ln (1-c)) / \lambda$.

Theorem 6.2. For all $n \in \mathbb{N}, L_{c}(n)<\gamma(c)$ c.

Proof. Let $r$ be the round in which the winner of the auction is found. The winner said yes in round $r$, so has a valuation at least equal to $p_{r}$. Since all other players say no in round $r$ or before, they have a valuation strictly less than $q_{r}$. The welfare loss is therefore bounded above by $q_{r}-p_{r}$, rand therefore by $\gamma(c)$. Hence, $L_{c}(n) \leqslant$ $\gamma(c) \cdot P_{c}(n)$. Applying the result of Theorem 6.1 completes the proof.

Many distributions have the feature that $\lim _{c \downarrow} \gamma(c)=0, r$ for instance the exponential distribution with parameter $\lambda$, rand any distribution with compact support like the uniform distribution. For such distributions, by choosing an appropriate fraction $c$ in the auction, we can limit the expected loss of welfare to an arbitrarily chosen level, independent of the number of players!

\subsection{Tradeoff between efficiency and running time}

From the analysis in this and the previous section, we derive the following relation between the value of $c$, the level of efficiency, and the running time. For a fixed number of players, a smaller fraction $c$ leads to a lower expected loss of welfare and a lower probability of inefficient allocation. But at the same time it leads to a higher expected number of rounds and queries. Thus, increasing running time is a price that we have to pay for increasing the level of welfare. Depending on the priorities of the auctioneer, he may trade off welfare against running time.

Fig. 6 shows the relationship between the expected running time and the probability of an inefficient allocation for several values of $n$. These relations are built on computational results based on the recursive formulas 2,4 , and 5 . Fig. 6(a) shows for every $n=5,10,20$, rand $30, \mathrm{r}$ a part of the curve $\left\{\left(e_{c}(n), P_{c}(n) \mid c \in(0,1)\right\}\right.$. In accordance with our bounds, it shows that for a fixed probability of inefficiency (vertical axis), we need a number of rounds (horizontal axis) that is logarithmically increasing in the number of bidders, using each time roughly the same $c$. Fig. 6(b) shows for every $n=5,10,20$ and 30 a part of the curve $\left\{\left(b_{c}(n), P_{c}(n) \mid c \in(0,1)\right\}\right.$. For a fixed probability of inefficiency, the number of rounds is now increasing linearly in the number of bidders. 
(a)

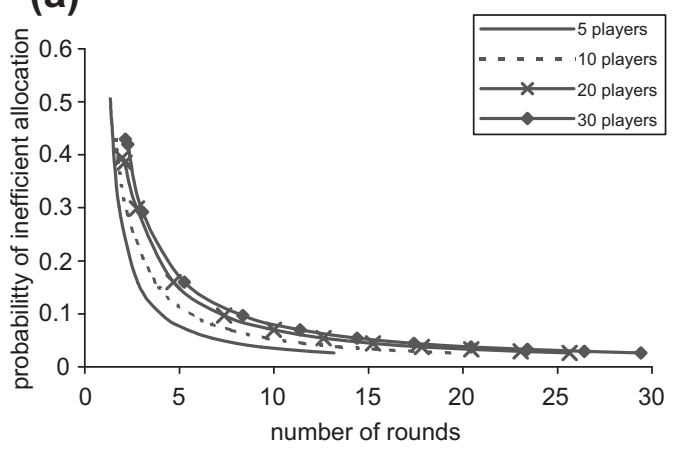

(b)

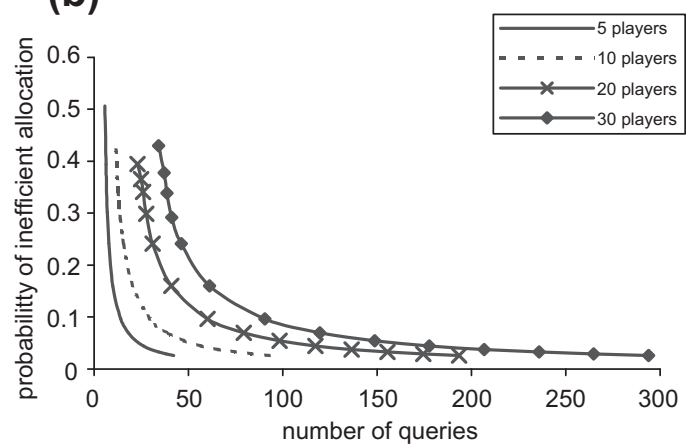

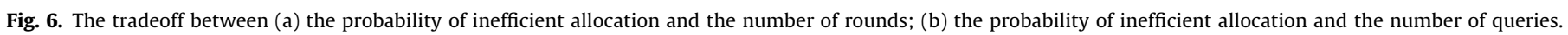

\section{Concluding remarks}

We have shown that $c$-fraction auctions provide an easy way of trading off efficiency versus running time of a single item auction. Bluff strategies form an ex-post equilibrium of these auctions. David et al. [9] proposed a slightly different ascending price query auction. Truthful reports to the queries form an ex-post equilibrium in that auction. We expect that choosing increments in their auction in the same way as they are chosen in the $c$-fraction auction provides similar bounds on the number of rounds, number of queries, and efficiency losses. Setting increments dynamically according to the $c$-fraction rule is thus an easy to implement method that leads to auctions that dominate rules-of-thumb approaches like fixed increments or fixed-percentage increments, as for example described in McAfee et al. [17], in all relevant dimensions.

\section{References}

[1] L. Ausubel, P. Cramton, P. Milgrom, The clock proxy auction: a practical combinatorial auction design, in: P. Cramton, Y. Shoham, R. Steinberg (Eds.), Combinatorial Auctions, MIT Press, 2006, pp. 116-138.

[2] L. Blumrosen, N. Nisan, I. Segal, Auctions with severely bounded communication, Journal of Artificial Intelligence Research 28 (2007) 233-266.

[3] L. Blumrosen, N. Nisan, I. Segal, Multi-player and multi-round auctions with severely bounded communication, in: Proceedings of 11th Annual European Symposium on Algorithms (ESA 03), 2003.

[4] M.S. Chwe, The discrete bid first auction, Economic Letters 31 (1989) 303-306.

[5] W. Conen, T. Sandholm, Preference elicitation in combinatorial auctions: extended abstract, in: Proceedings of the ACM Conference on Electronic Commerce (ACM-EC), 2001, pp. 256-259.

[6] V. Conitzer, T. Sandholm, Computational criticisms of the revelation principle, in: Proceedings of the AAMAS-03 Workshop on Agent Mediated Electronic Commerce V (AMEC V), 2003.

[7] P. Cramton, Simultaneous ascending auctions, in: P. Cramton, Y. Shoham, R Steinberg (Eds.), Combinatorial Auctions, MIT Press, 2004, pp. 99-115.

[8] E. David, A. Rogers, J. Schiff, S. Kraus, N.R. Jennings, Optimal design of English auctions with discrete bid levels, in: Proceedings of ACM Conference on Electronic Commerce (EC'05), 2005, pp. 98-107.
[9] E. David, A. Rogers, N.R. Jennings, J. Schiff, S. Kraus, M.H. Rothkopf, Optimal design of english auctions with discrete bid levels, ACM Transactions on Internet Technology 7 (2007). Article 12.

[10] S. de Vries, J. Schummer, R.V. Vohra, On ascending Vickrey auctions for heterogeneous objects, Journal of Economic Theory 132 (2007) 95-118.

[11] R. Engelbrecht-Wiggans, C.M. Kahn, Protecting the winner: second-price versus oral auctions, Economics Letters 35 (1991) 243-248.

[12] E. Grigorieva, P.J.J. Herings, R.M. üller, D. Vermeulen, The communication complexity of private value single item auctions, Operations Research Letters 34 (2006) 491-498.

[13] E. Grigorieva, P.J.J. Herings, R.M. üller, D. Vermeulen, The private value single item bisection auction, Economic Theory 30 (2007) 107-118.

[14] E. Grigorieva, P.J.J. Herings, R.M. üller, D. Vermeulen, Inefficiency of equilibria in digital mechanisms with continuous valuations, Journal of Mathematical Economics 47 (2011) 541-544.

[15] B. Hudson, T. Sandholm, Effectiveness of preference elicitation in combinatorial auctions, in: Proceedings of the Agent-Mediated Electronic Commerce (AMEC) Workshop at AAMAS-02, 2004.

[16] K. Larson, T. Sandholm, costly valuation computation in auctions, Proceedings of the Theoretical Aspects of Reasoning about Knowledge (TARK) (2001) 169-182.

[17] R.P. McAfee, J. McMillan, S. Wilkie, The greatest auction in History, in: J. Siegfried (Ed.), Better Living Through Economics, Harvard University Press, 2010, pp. 168-187.

[18] D. Mishra, D. Veeramani, Vickrey-Dutch procurement auction for multiple items, European Journal of Operational Research 180 (2007) 617-629.

[19] N. Nisan, I. Segal, The communication requirements of efficient allocations and supporting prices, Journal of Economic Theory 129 (2006) 192-224.

[20] D. Parkes, Optimal auction design for agents with hard valuation problems, in: Proceedings of IJCAI-99 Workshop on Agent Mediated Electronic Commerce, 1999, pp. 206-219.

[21] M.H. Rothkopf, T.J. Tisberg, E.P. Kahn, Why are Vickrey auctions rare?, Journal of Political Economy 98 (1990) 94-109

[22] M.H. Rothkopf, R.M. Harstad, On the role of discrete bid levels in oral auctions, European Journal of Operational Research 74 (1994) 572-581.

[23] T. Sandholm, Issues in computational Vickrey auctions, International Journal of Electronic Commerce 4 (2000) 107-129.

[24] T. Sandholm, C. Boutilier, Preference elicitation in combinatorial auctions, in: P. Cramton, Y. Shoham, R. Steinberg (Eds.), Combinatorial Auctions, MIT Press, 2006, pp. 233-264

[25] W. Vickrey, Counterspeculation, auctions and competitive sealed tenders, Journal of Finance 16 (1961) 8-37.

[26] J. Yu. Discrete approximation of continous allocation mechanisms, Ph.D. Thesis, Division of the Humanities and Social Sciences, California Institute of Technology, 1999. 